\title{
Fisiologia Fractal: a dimensão de Higuchi e suas aplicações
}

\author{
$\underline{\text { Lara Martins Barbosa* }}{ }^{*} \quad$ Ana Maria Amarillo Bertone \\ Anielle G. Vaz Coelho \\ Faculdade de Matemática, UFU, \\ 38408-100, Uberlândia, MG \\ E-mail: lara-barbosa@hotmail.com, anamaria@famat.ufu.br, aniele_vaz@hotmail.com
}

\section{RESUMO}

Este trabalho é o resultado da primeira etapa de um projeto sobre o estudo da Fisiologia Fractal, metodologia que se foca na complexidade do corpo humano, dando uma caracterização desta complexidade através das medidas fractais. Em particular para este projeto, foi aprofundado o conceito de Dimensão fractal de Higuchi $[4,5]$.

Uma das explicações para o comportamento irregular observado nos sistemas dinâmicos que governam os processos de origem biológico, é fornecido por uma das áreas matemáticas mais marcantes das últimas décadas: a teoria do caos. Um dos aspectos mais sugestivos da teoria do caos determinístico é o conceito de geometria fractal, caracterizada pela autossemelhança e pela dimensão não inteira.

Em pesquisas recentes (como exemplo [2]), as experiências mostram a tendência de reconhecer que as estruturas anatômicas do corpo humano, como por exemplo a superfície convoluta do cérebro ou a complexidade das redes neurais, são, especialmente na sua dinâmica, objetos fractais, da mesma natureza que àqueles descritos pelo "pai" da teoria fractal, como é conhecido o matemático Benoit Mandelbrot [1] que deu nome a esses tipos de objetos matemáticos.

A grande diferença de usar esta metodologia de interpretação dos fenômenos biológicos, se baseia no fato que a dimensão fractal acaba sendo significativamente um melhor indicador do estado das funções organísmicas na saúde e na doença do que as medidas "tradicionais", tais como a frequência dos sinais de um eletroencefalograma, a frequência cardíaca ou respiratória.

A observação de que a fisiologia humana é essencialmente fractal foi feita pela primeira vez na década dos anos 80 , com base na análise de um número limitado de conjuntos de dados. Foi no começo deste século que as pesquisas foram intensificadas, resultando em um melhor apuramento dos diagnósticos e delimitação da fronteira entre os casos de quadro sadio e doente.

É mostrado em [2] que a dimensão fractal de Higuchi é uma ferramenta poderosa na análise da organização macro estrutural dos sinais de um eletroencefalograma, por exemplo no resultado de distinguir o autismo do retardo mental. Os resultados da análise fractal revelam que as crianças autistas produzem no eletroencefalograma séries temporais mais complexas que a dos deficientes mentais. Com esta motivação, foi desenvolvido o estudo da dimensão fractal de Higuchi e uma aplicação do algoritmo para uma série temporal simulada a partir de dados reais.

O algoritmo de Higuchi consiste em considerar $n$ pontos $x(1), x(2), \ldots, x(N)$ de uma série temporal de dados e construir a partir dela uma outra série temporal da forma:

$$
x_{k}^{k}=x(m), x(m+k), x(m+2 k), \ldots, x\left(m+\left\lfloor\frac{N-m}{k} k\right\rfloor\right) m=1,2, \ldots, k, k \in \mathbb{N}-\{0\},
$$

onde $m$ representa o tempo inicial e $k \in[1, k m a x]$ indica o tempo de retardo e o símbolo $\lfloor\cdot\rfloor$ indica a parte inteira do número dado. Para cada $x_{k}^{m}$ contruímos uma série de comprimento

${ }^{*}$ Discente do PETMAT - SESu/MEC 
$L_{m}(k)$, é calculada como

$$
L m(k)=\frac{k(N-1)}{\left\lfloor\frac{N-m}{k}\right\rfloor}\left(\sum_{i=1}^{\left\lfloor\frac{N-m}{k}\right\rfloor} \mid x(m+i k)-x(m+(i-1) k) j\right),
$$

onde o termo $\frac{N-1}{\left\lfloor\frac{N-m}{k}\right\rfloor}$ é um fator normalizante. A média do comprimento é calculada para todos os tempos da série para o mesmo retardo $k$ assim como a média dos comprimentos $L_{m}(k)$ para $m=1,2, \ldots, k$. Este procedimento é repetido para cada $k$ variando no intervalo de validação de $[1, k \max ]$. No caso de existir o limite $k^{H}$ quando $m \rightarrow \infty$, então a série temporal $x$ é fractal e tem como dimensão $H$, chamada de dimensão de Higuchi. Assim, representando linearmente no domínio $1 / k$, a inclinação da reta de ajuste dos dados representa a estimativa de Higuchi da dimensão fractal, de forma análoga, que é considerada a dimensão de Haussdoff.

A segunda etapa deste projeto, já em andamento, tem como objetivo aplicar esta metodologia a séries temporais obtidas a partir de banco de dados reais, resultados de pacientes saudáveis em comparação com pacientes com problemas coronários, sendo que os dados são obtidos de pacientes do hospital universitário da Universidade Federal de Uberlândia. Para o momento da realização do CNMAC 2014, as autoras pretendem mostrar também os resultados desta segunda etapa.

Palavras-chave: Fisiologia Fractal, Cálculo Fracionário, Dimensão de Higuchi

\section{Referências}

[1] B. Mandelbrot The Fractal Geometry of Nature. New York W. H. Freeman and Co., 1982.

[2] W. Klonowski, E. Olejarczyk , R. Stepien, Nonlinear Dynamics Algorithms for Time Series Analysis -Implementation in EEG Data, em "Attractors, Signals, and Synergetics, Frontiers on Nonlinear Dynamics"', Pabst Science Publishers, 1, pp. 553-560, 2002.

[3] W. Klonowski, From conformons to human brains: an informal overview of nonlinear dynamics and its applications in biomedicine em "Nonlinear Biomed Phys", 1 (5) 2007.

[4] T. Higuchi, "Relationship between the fractal dimension and the power law index for a time series: A numerical investigation," Physica D, 46, pp. 254-264, 1990.

[5] T. Higuchi, "Approach to an irregular time series on the basis of the fractal theory" Physica D, 31, 2, pp. 277-283, 1988. 